\title{
Altered aortic 3D hemodynamics and geometry in pediatric Marfan syndrome patients
}

Roel L. F. van der Palen ${ }^{1,2^{*}}$, Alex J. Barker ${ }^{1}$, Emilie Bollache ${ }^{1}$, Julio Garcia ${ }^{1,3}$, Michael J. Rose ${ }^{4}$, Pim van Ooij, ${ }^{1,5}$, Luciana T. Young ${ }^{7}$, Arno A. W. Roest ${ }^{2}$, Michael Markl ${ }^{1,6}$, Joshua D. Robinson ${ }^{1,7,8}$ and Cynthia K. Rigsby ${ }^{1,4,8}$

\begin{abstract}
Background: Blood flow dynamics make it possible to better understand the development of aortopathy and cardiovascular events in patients with Marfan syndrome (MFS). Aortic 3D blood flow characteristics were investigated in relation to aortic geometry in children and adolescents with MFS.

Methods: Twenty-five MFS patients (age $15.6 \pm 4.0$ years; 11 females) and 21 healthy controls (age $16.0 \pm 2.6$ years; 12 females) underwent magnetic resonance angiography and 4D flow CMR for assessment of thoracic aortic size and 3D blood flow velocities. Data analysis included calculation of aortic diameter and BSA-indexed aortic dimensions (Z-score) along the thoracic aorta, 3D mean systolic wall shear stress (WSS mean) in ten aortic segments and assessment of aortic blood flow patterns.

Results: Aortic root (root), ascending (AAo) and descending (DAo) aortic size was significantly larger in MFS patients than healthy controls (Root Z-score: $3.56 \pm 1.45$ vs $0.49 \pm 0.78, p<0.001$; AAo Z-score $0.21 \pm 0.95$ vs $-0.54 \pm 0.64, p=0$. 004; proximal DAo Z-score $2.02 \pm 1.60$ vs $0.56 \pm 0.66, p<0.001)$. A regional variation in prevalence and severity of flow patterns (vortex and helix flow patterns) was observed, with the aortic root and the proximal DAo (pDAo) being more frequently affected in MFS. MFS patients had significantly reduced WSS mean in the proximal AAo (pAAo) outer segment ( $0.65 \pm 0.12$ vs. $0.73 \pm 0.14 \mathrm{~Pa}, p=0.029)$ and $\mathrm{pDAo}$ inner segment $(0.74 \pm 0.17 \mathrm{vs} .0 .87 \pm 0.21 \mathrm{~Pa}, p=0.021)$, as well as higher WSS $_{\text {mean }}$ in the inner segment of the distal AAo $(0.94 \pm 0.14 \mathrm{vs}$. $0.84 \pm 0.15 \mathrm{~Pa}, p=0.036)$ compared to healthy subjects. An inverse relationship existed between pDAo WSS mean and both pDAo diameter $(R=-0.53, p<0.001)$ and \% diameter change along the pDAo segment $(R=-0.64, p<0.001)$.

Conclusions: MFS children and young adults have altered aortic flow patterns and differences in aortic WSS that were most pronounced in the pAAo and pDAo, segments where aortic dissection or rupture often originate. The presence of vortex flow patterns and abnormal WSS correlated with regional size of the pDAo and are potentially valuable additional markers of disease severity.
\end{abstract}

Keywords: Marfan syndrome, Aorta hemodynamics, Aortic geometry, 4D flow, Children

\footnotetext{
*Correspondence: r.vanderpalen@lumc.nl

'Department of Radiology, Feinberg School of Medicine, Northwestern

University, Chicago, IL, USA

2Division of Pediatric Cardiology, Department of Pediatrics, Leiden University

Medical Center, Albinusdreef 2, 2333, ZA, Leiden, The Netherlands

Full list of author information is available at the end of the article
} 


\section{Background}

Marfan syndrome (MFS) is an inherited autosomal dominant connective tissue disease, mostly related to mutations in the fibrillin-1 (FBN1) gene. Many organ systems can be involved, but most life-threatening complications are related to the cardiovascular system and include aortic dissection and aortic rupture. Although the entire aorta may dilate in MFS, specific aortic regions are prone for progressive dilation and dissection and represent the aortic root and the proximal descending aorta (pDAo) [1-3]. Before the implementation of preventive surgical management strategies, two-thirds of dissections and ruptures occurred in the ascending aorta (AAo) while one-third occurred in the descending aorta (DAo). Recently, this ratio has shifted towards proportionally more DAo complications $[4,5]$. Thus, pathology of the aorta distal to the aortic root remains a cause of concern.

In the current guidelines, absolute aortic diameters and identification of changes in aortic dimension play an important role in risk stratification and decision making for preventive surgery [6]. However, it is known that aortic dissection may occur in only moderately dilated aortas, and recent literature has shown that only about $50 \%$ of MFS patients with pDAo dissections had pDAo diameters larger than the upper limit of normal [5]. These findings indicate that aortic dimension alone may not capture the predictive risk for adverse cardiovascular events.

It is not well known how aortic hemodynamics interact with the altered vascular structure of the aorta in MFS. Independent associations between hemodynamic markers and aortopathy have been presented in cardiovascular magnetic resonance (CMR) imaging studies [7-10]. These studies employed time-resolved 3D phase-contrast CMR with three-directional velocity encoding (4D flow CMR) to enable a comprehensive, non-invasive in-vivo investigation of cardiac and aortic hemodynamics. The technique allows for quantitative analysis of advanced hemodynamic parameters [11-14] such as wall shear stress (WSS), which is defined as the tangential force exerted by blood flow on the aortic wall. WSS has recently been correlated with evidence of molecular and architectural medial wall dysfunction in bicuspid aortic valve-related aortopathy [15]. WSS may also be a useful parameter to identify focal regions with wall dysfunction in MFS patients. The aim of the study was to investigate regional aortic WSS, flow patterns and aortic dimensions in a pediatric MFS cohort compared to a healthy age appropriate control cohort by using a novel 3D WSS technique.

\section{Methods}

\section{Study population}

Twenty-five patients with MFS (mean age $15.6 \pm 4.0$ years, 11 females) were included in this Institutional Review Board (IRB) approved and HIPAA compliant study. All patients underwent 4D flow CMR immediately following a clinically ordered standard-of-care CMR assessment of the entire aorta. The diagnosis of MFS was based on clinical criteria according to the international standards derived from the 2010 Revised Ghent Criteria [16]. In addition to the clinical criteria, conventional genetic testing for FBN1 mutation was performed in 23 MFS patients; in 22 of them a FBN1 mutation was proven. Patients with a bicuspid aortic valve or prior aortic surgery were excluded. Twenty-one healthy pediatric subjects (mean age $16.0 \pm 2.6$ years, 12 females) with a tricuspid aortic valve without history of cardiovascular disease and normal cardiac and aortic valve function, who underwent 4D flow CMR following a standard-of-care CMR examination, were collected from a retrospective chart review as approved by the IRB and were used as a healthy control group. The majority of these subjects underwent CMR for atypical chest pain after the inability of echocardiography to detect the origins of coronary arteries. The CMR revealed no morphological and functional cardiac or large vessel abnormalities in any of the healthy subjects. Written informed consent was obtained from all participants or their legal guardians for the addition of the 4D flow CMR to the standardof-care CMR protocol.

\section{Magnetic resonance imaging}

CMR examinations were performed on $1.5 \mathrm{~T}$ systems (MAGNETOM Avanto or Area, Siemens, Germany). All patients and healthy subjects underwent a standard-ofcare CMR exam that included dynamic ECG gated twodimensional (2D) cine steady state free precession (SSFP) imaging, for the evaluation of cardiac anatomy and function, as well as ECG gated and navigator triggered time-resolved contrast-enhanced MR angiography (CE-MRA) after administration of blood pool contrast media (gadofosveset trisodium, ABLAVAR, $0.03 \mathrm{mmol} /$ $\mathrm{kg}$, Lantheus, N. Billerica, MA), for evaluation of aortic morphology and dimensions. Sequence parameters for CE-MRA: spatial resolution: $1.3-1.5 \times 1.3-1.5 \times 1.3$ $-1.7 \mathrm{~mm}^{3}$, echo time/repetition time: $1.2-1.6 \mathrm{~ms} / 3.2-$ $3.6 \mathrm{~ms}$, flip angle: $18^{\circ}$. For assessment of aortic blood flow, 4D flow CMR was acquired during free breathing using respiratory navigator and prospective ECG-gating, with full volumetric coverage of the thoracic aorta in an oblique sagittal orientation. Standardized 4D flow sequence parameters were used throughout the study for age categories: $6-12$ years and $>12$ years of age. Average 4D flow scan parameters were as follows: spatial resolution: $2.3-3.8 \times 1.6-2.0 \times 1.8-3.0 \mathrm{~mm}^{3}$, temporal resolution: $38.4-41.6 \mathrm{~ms}$, echo time/repetition time: $2.4-2.7 \mathrm{~ms} / 5.0-5.1 \mathrm{~ms}$, flip angle: $15^{\circ}$, field of view: 250 $-320 \times 141-250 \mathrm{~mm}^{2}$, matrix size: $160 \times 70-130$ and velocity sensitivity: $120-200 \mathrm{~cm} / \mathrm{s}$. 


\section{Data analysis}

\section{Thoracic aortic size}

Thoracic aortic diameters were determined from the CE-MRA exams using a 3D workstation with multiplanar reformatting capabilities (Vitrea, Vital Images, Minneapolis, MN). According to the international guidelines [17], maximum orthogonal aortic diameters were measured along the aorta from inner-edge to inner-edge at the following levels: STJ: sinotubular junction; midAAo: mid-ascending aorta, at the level of the right pulmonary artery; dAAo: distal ascending aorta, prior to innominate artery; distal aortic arch, between left carotid artery and left subclavian artery; aortic isthmus, at the level direct beyond the left subclavian artery; pDAo: proximal descending aorta, at the level of the ductal ampulla; midDAo: mid-descending aorta, at the level of the left atrium; dDAo: distal descending aorta, at the level of the diaphragm (Fig. 1b).

Maximum aortic root (Root) diameter was measured on a short-axis cine SSFP plane across the aortic root at peak systole [18, 19]. Change in aortic diameter along aortic segments was calculated and expressed as percentage change for the segments aortic root to midAAo and aortic isthmus to pDAo: $\left(\frac{\mid \text { Aortic root-midAAo } \mid}{\text { Aortic root }}\right) * 100$ and $\left(\frac{\mid \text { Aortic isthmus- } p D A o \mid}{\text { Aortic isthmus }}\right) * 100$. To account for the range of patient age and body size, aortic Z-scores were calculated for each patient from the CMR aortic measurements (AAo through pDAo) and the body surface area (BSA; determined according to the method of Mosteller) [20], using EchoIMS (Merge Healthcare, Chicago, IL). The Z-score is a nomogram-based metric for assessing aortic dilatation in pediatric patients in which a Z-score between -2 and
+2 is considered normal [21]. While EchoIMS provides ultrasound derived normative data, there is currently no CMR-specific database, and as such, this method of normalization is the best available alternative and supported by previous studies [22, 23]. Particularly, Z-scores of the pDAo were calculated from the data on the aortic isthmus region of EchoIMS data. An aortic Z-score $\geq 2.0$ indicates that the aortic diameter at that level is outside the upper normal range ( $\geq 2$ standard deviations from the mean). Based on pDAo Z-scores, MFS patients were divided into subgroups for additional analysis: group 1: MFS Z-score pDAo 2 2.0; group 2: MFS Z-score pDAo $<2.0$.

\section{D flow data processing and $3 D$ blood flow visualization}

All 4D flow CMR data (Fig. 1a) were corrected for noise, velocity aliasing, Maxwell terms and eddy currents using Matlab-based in-house software (MathWorks, Natick, MA) as described previously [24]. A 3D phase-contrast MR angiogram was created from the corrected 4D flow CMR data and was used to semi-automatically define a 3D segmentation of the thoracic aorta (Mimics, Materialise, Leuven, Belgium) (Fig. 1b). The 3D segmentation was used to mask the velocity field for the generation of time-resolved 3D pathlines to visualize aortic 3D blood flow (Ensight, CEI, Apex, NC). Semiquantitative aortic flow pattern analysis using pathline movies was performed in MFS patients and healthy subjects in a blinded fashion and random order by two observers (CR and JR). A helical flow pattern was defined as rotational fluid motion around an axis parallel to the bulk fluid motion (i.e. along the longitudinal axis of the vessel). Vortical flow patterns were defined as revolving particles around a point within the vessel with a rotation direction deviating by $>90^{\circ}$ from the physiological flow direction.

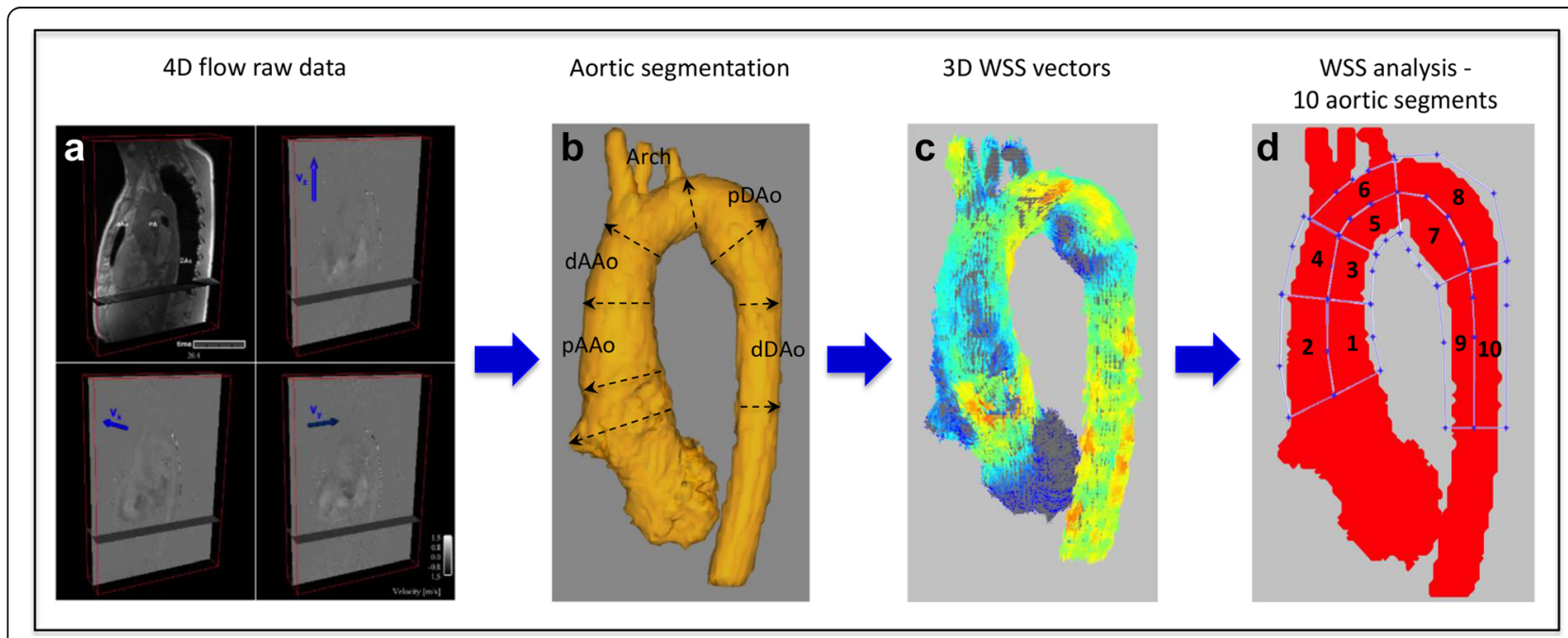

Fig. 1 Aortic 4D flow data processing and analysis. a 4D flow raw data including anatomical and flow data. b volumetric aorta segmentation; levels of aortic diameter measurements. c calculation of systolic 3D WSS vector maps. $\mathbf{d}$ regional WSS analysis in ten aortic regions 
Vortex and helix flow pattern grading was assessed in three aortic segments (AAo, Arch and DAo) on a 3-point ordinal scale: 0 : no vortex/helix patterns; 1 : flow rotation $<360^{\circ}$; 2 : flow rotation $>360^{\circ}$ (Examples in Fig. 2).

\section{Regional wall shear stress assessment}

Based on the 3D segmentation, 3D mean systolic wall shear stress $\left(\mathrm{WSS}_{\text {mean }}\right.$ ) along the entire aortic vessel wall was calculated from 4D flow velocity data (Fig. 1c) using a previously described algorithm, which was shown to provide good reproducibility $[25,26]$. Briefly, WSS vector was estimated for each wall point on the aortic surface based on the 3D velocity spatial gradient perpendicular to the vessel wall. Systolic WSS vector magnitude was defined as the average over five cardiac time frames centered on the peak systolic time frame (i.e., peak systolic phase \pm 2 phases). WSS $_{\text {mean }}$ was calculated within ten aortic regions that were manually drawn based on anatomic landmarks (Fig. 1d): 1) inner and outer proximal ascending aorta (pAAo, from STJ to midAAo); 2) inner and outer distal ascending aorta (dAAo, from midAAo to the origin of the innominate artery); 3) inner and outer aortic arch (from origin innominate artery until left subclavian artery); 4) inner and outer proximal descending aorta (pDAo, beyond the left subclavian artery to mid-descending thoracic aorta); 5) inner and outer distal descending aorta (dDAo, from middescending thoracic aorta to the level of the aortic valve). Of note, the supra-aortic arch branches were excluded from the regional WSS measurements.

\section{Statistical analysis}

Statistical analysis was performed using IBM SPSS Statistics 20.0 (SPSS Inc., Chicago, IL, USA). Baseline characteristics are provided as mean \pm standard deviation for continuous variables and percentage for discrete variables. Shapiro-Wilk tests were performed to test for equal distribution of continuous variables. A chi-square test was used to investigate differences in gender distribution between the MFS and healthy subject groups. A non-paired $t$-test was performed to study differences in continuous variables from baseline characteristics between the two cohorts. For comparison of differences between aortic Z-scores as well as regional aortic WSS between the two cohorts a Mann Whitney test was used. A Kruskal-Wallis test was used to evaluate differences in aortic Z-score, size change and regional WSS between the two MFS subgroups, as well as between the MFS subgroup with a pDAo Z-score $\geq 2.0$ and healthy subjects. The relationships between aortic dimensions and

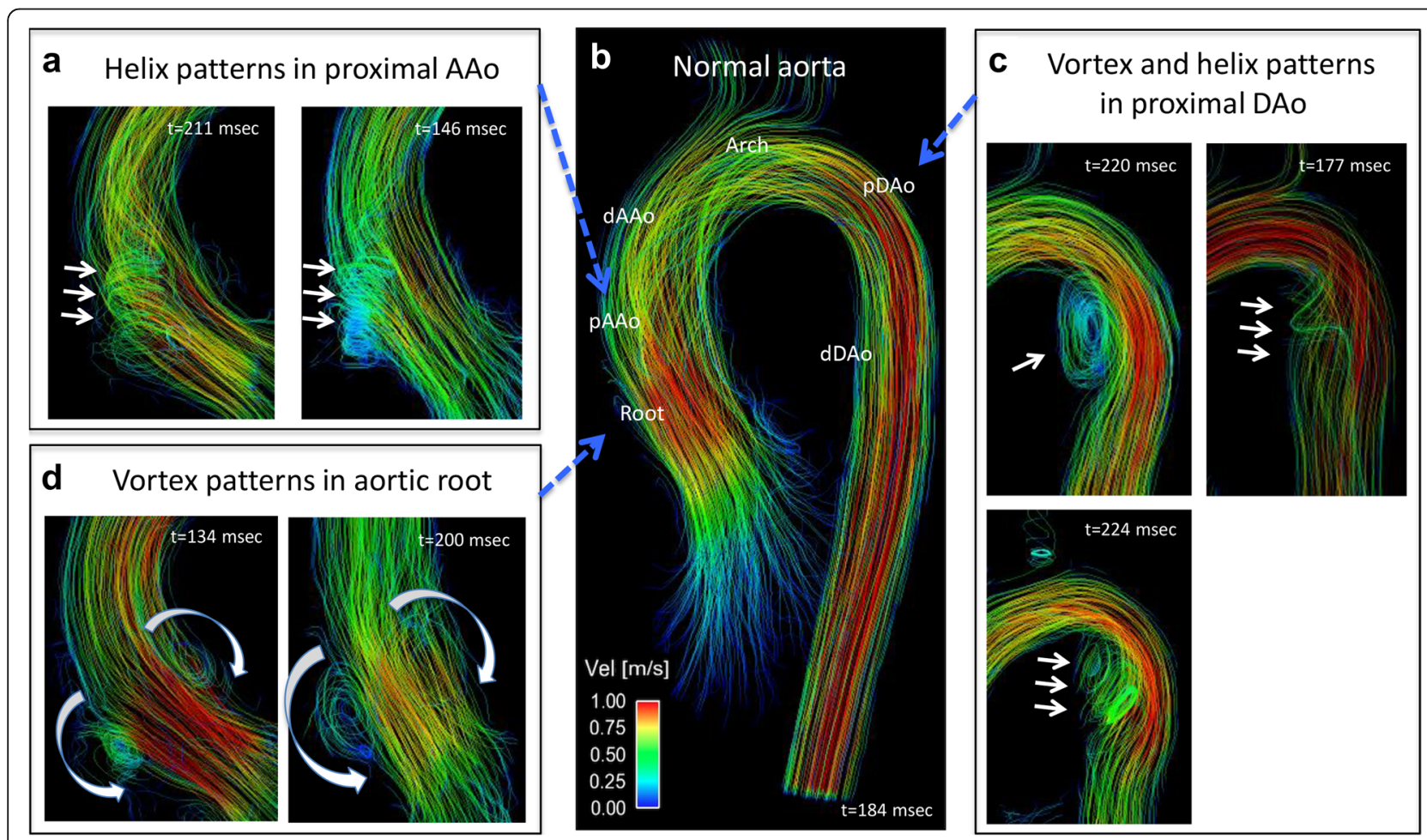

Fig. 2 Aortic 3D blood flow characteristics (systolic streamlines). Panel b represents systolic streamline visualization of a normal aorta from a healthy control. Panels $\mathbf{a}, \mathbf{c}$ and $\mathbf{d}$ illustrate typical secondary flow patterns in Marfan syndrome patients during systole, like a helix flow pattern from the aortic root into the pAAo (a, grade 2) and vortex and helix flow patterns in the pDAo (c, grade 2). $\mathbf{d}$ prominent vortex flow patterns in the aortic root 
hemodynamic parameters were investigated using correlation analysis based on linear regression. A $p$-value $<$ 0.05 was considered statistically significant for all statistical tests. Inter-observer agreement on qualitative assessment of helix and vortex flow formation between two observers was calculated using Cohen's $\kappa$ statistics. A $K$ value of $0.61-0.80$ corresponded to substantial interobserver agreement; values of $0.41-0.60$ corresponded to moderate agreement.

\section{Results}

Patient characteristics are summarized in Table 1. As expected, MFS patients were typically taller than healthy subjects. All patients and healthy subjects had normal systolic biventricular function and no significant regurgitation of the mitral valve or aortic valve, except for one MFS patient who showed moderate mitral valve regurgitation.

Table 1 Baseline characteristics

\begin{tabular}{llll}
\hline & Marfan & Healthy controls & $p$-value \\
\hline N (females) & $25(11)$ & $21(12)$ & 0.375 \\
Age $(\mathrm{y})$ & $15.6 \pm 4.0$ & $16.0 \pm 2.6$ & 0.720 \\
Height $(\mathrm{cm})$ & $178.6 \pm 17.3$ & $161.2 \pm 11.2$ & $<0.001^{*}$ \\
Weight $(\mathrm{kg})$ & $65.6 \pm 20.2$ & $58.8 \pm 15.1$ & 0.208 \\
BSA $\left(\mathrm{m}^{2}\right)$ & $1.78 \pm 0.36$ & $1.61 \pm 0.25$ & 0.082 \\
BMI $\left(\mathrm{kg} / \mathrm{m}^{2}\right)$ & $20.1 \pm 4.5$ & $22.4 \pm 4.0$ & 0.081 \\
LV ejection fraction $(\%)$ & $55.7 \pm 6.5$ & $58.1 \pm 3.7$ & 0.243 \\
Bilateral ectopia lentis $(n, \%)$ & $5(20 \%)$ & 0 &
\end{tabular}

Genetic mutation ( $n$ )

$\begin{array}{lll}\text { FBN1 gene } & 22 & - \\ \text { Unknown } & 3 & - \\ & 20(80 \%) & 0 \\ \text { Cardiac medication, }(n, \%) & \\ \text { 1. Beta-blocker } & 15(60 \%) & - \\ \text { 2. AT II-receptor antagonist } & 7(28 \%) & - \\ \text { 3. ACE inhibitor } & 3(12 \%) & - \\ \text { 4. Combination } 1+2 & 4(16 \%) & - \\ \text { 5. Combination } 1+3 & 1(4 \%) & -\end{array}$

Thoracic aortic size (Z-score)

$\begin{array}{llll}\text { Aortic root } & 3.56 \pm 1.45 & 0.49 \pm 0.78 & <0.001^{*} \\ \text { ST-junction } & 1.70 \pm 1.28 & -0.34 \pm 0.77 & <0.001^{*} \\ \text { Ascending aorta } & 0.21 \pm 0.95 & -0.54 \pm 0.64 & 0.004^{*} \\ \text { Distal aortic arch } & 0.14 \pm 0.90 & -0.23 \pm 0.66 & 0.155 \\ \text { Aortic isthmus } & 0.96 \pm 0.94 & 0.43 \pm 0.49 & 0.039^{*} \\ \text { Proximal descending aorta } & 2.02 \pm 1.60 & 0.56 \pm 0.66 & <0.001^{*}\end{array}$

The $p$ value stems from non-paired t-test, for sex from Chi-square test and for thoracic aorta dimensions from the Mann Whitney test, ${ }^{*} p<0.05$. Abbreviations: $A C E$ angiotensin converting enzyme, $A T$ angiotensin, $B M I$ body mass index, $B S A$ body surface area, FBN1 fibrillin-1, LV left ventricular, ST sinotubular

\section{Thoracic aorta dimensions}

As summarized in Table 1, the aortic diameter Z-scores were significantly greater in MFS patients compared to healthy subjects at all thoracic aorta levels $(p \leq 0.05)$, except for the distal aortic arch $(p=0.155)$. Aortic dilatation in the MFS population was most prominent in the aortic root and pDAo, with Z-scores of $3.56 \pm 1.45$ and $2.02 \pm 1.60$, respectively. Using a Z-score $\geq 2.0$ to define aortic dilatation, $n=21$ (84\%) MFS patients had aortic root dilatation and $n=10(40 \%)$ had dilatation of the STJ. Seven MFS patients (28\%) had a pDAo Z-score $\geq 2.0$.

\section{Aortic flow patterns}

There was moderate to substantial inter-observer agreement for grading of flow patterns within the AAo (Kappa 0.54) and DAo (Kappa 0.84) between the two observers. Examples of 3D blood flow patterns found in MFS patients are depicted in Fig. 2 (Panels a, c and d) and Additonal file 1: Video S1. Marked vortex flow patterns were noted in the pDAo region of 14 MFS patients and in only two healthy subjects (56\% vs. $9.5 \%$ of total subjects, $p=0.002$ ), with an average $\mathrm{pDAo}$ vortex grading significantly higher in MFS patients than healthy subjects $(0.82 \pm 0.83$ vs. $0.14 \pm 0.39, p<0.001)$. These vortex flow patterns were most pronounced in MFS patients with a dilated pDAo (6 out of 7) (Fig. 3; Additional file 1: Video S1). Physiological vortex patterns in the sinuses of the aortic root were more often visible in MFS patients compared to healthy subjects $(76.0 \%$ vs. $23.8 \%$, $p<0.001$; Fig. 2, Panel d; Additional files 1 and 2: Video S1 and S2). Non-physiological helical flow patterns with high strength (grade 2) originating from the aortic root were observed in $16 \%$ of the MFS patients and were not present in any of the healthy subjects $(p<0.05$, Fig. 2, Panel a). Finally, throughout the AAo, no difference in presence or strength of major flow patterns was observed between the MFS patients and healthy subjects (MFS vs. healthy subjects: prevalence $76.0 \%$ vs. $57.1 \%, p=0.17$; average helix grading $1.18 \pm 0.71$ vs. $0.86 \pm 0.76, p=0.14$ ). No helix or vortex flow patterns were present in the aortic arch for both MFS patients and healthy subjects.

\section{Segmental aortic WSS \& correlations with diameter}

Figure 4a illustrates regional differences in $\mathrm{WSS}_{\text {mean }}$ between MFS patients and healthy subjects. MFS patients showed reduced WSS $_{\text {mean }}$ in the pAAo outer segment $(0.65$ \pm 0.12 vs. $0.73 \pm 0.14 \mathrm{~Pa}, p=0.029)$ and $\mathrm{pDAo}$ inner segment $(0.74 \pm 0.17$ vs. $0.87 \pm 0.21 \mathrm{~Pa}, p=0.021)$ compared to healthy subjects (Fig. 4a). There was a higher WSS $_{\text {mean }}$ in the inner segment of the dAAo in MFS patients $(0.94 \pm 0.14$ vs. $0.84 \pm 0.15 \mathrm{~Pa}, p=0.036)$. Moreover, in the entire study cohort (MFS and healthy subjects), WSS mean $_{\text {showed a }}$ strong inverse relationship with aortic dimensions, as well as with diameter changes along aortic segments. The most 


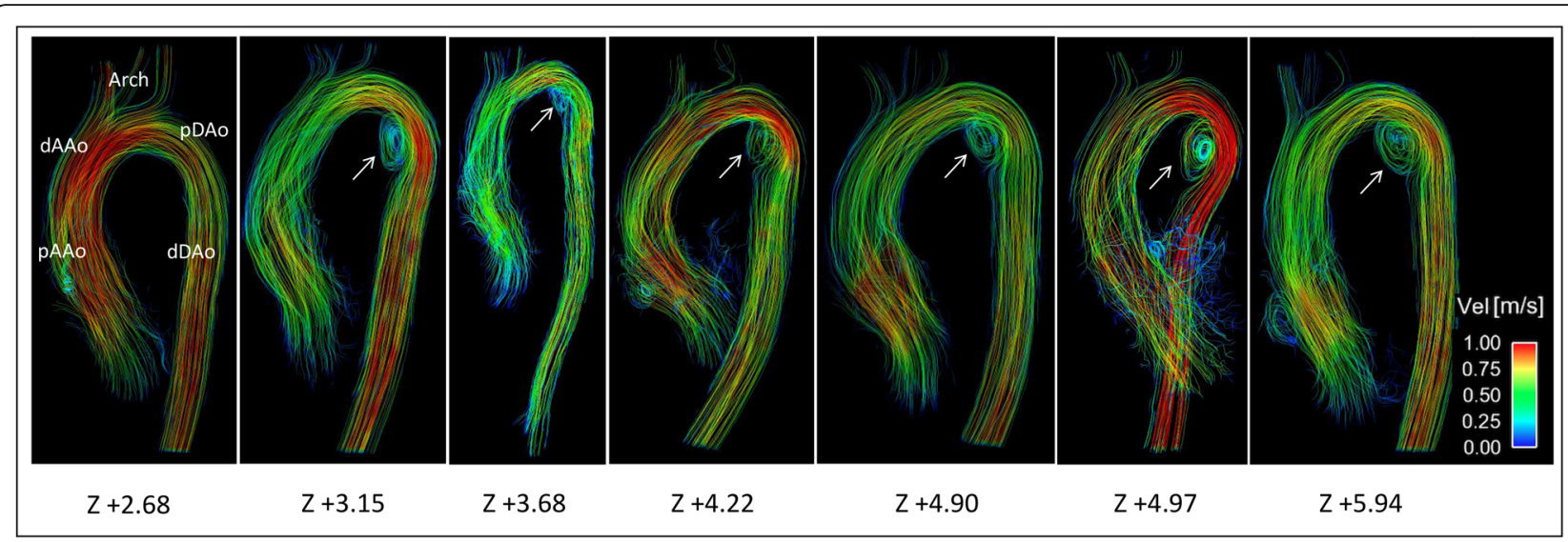

Fig. 3 Aortic blood flow streamlines in MFS patients with Z-score $\geq 2.0$ of the proximal descending aorta $(n=7)$. Local vortex flow patterns at the inner curvature of the pDAo (arrows) in six out of the seven MFS patients with dilated pDAo (Z-score $\geq 2.0$ )

prominent association was found between the $\mathrm{WSS}_{\text {mean }}$ of the pDAo inner segment and both the pDAo Z-score $(R=-0.53, p<0.001)$ and the diameter change along the corresponding segment $(R=-0.64, p<0.001$; Fig. 5$)$.

\section{Subgroup analysis according to proximal DAo dilatation} Based on pDAo Z-scores, MFS patients were divided into subgroups for additional analysis. Seven MFS patients had a Z-score pDAo $\geq 2.0$ (group 1), whereas 18 MFS patients had a Z-score pDAo $<2.0$ (group 2). Age, gender and BSA were comparable between the MFS subgroups with dilated pDAo (Z-score $\geq 2.0$ ) and healthy subjects (Table 2). Differences in aortic diameter and segmental WSS between healthy subjects and MFS subgroups as well as between the two MFS subgroups are summarized in Table 2. Dimensions of the entire thoracic aorta in the MFS subgroup with dilated pDAo showed significantly greater Z-scores compared to both other groups, except for the aortic root (Table 2). MFS patients with a dilated pDAo showed significantly lower $\mathrm{WSS}_{\text {mean }}$ in the inner pDAo aortic segment $(p<0.05)$ compared to both the MFS patients with pDAo Z-score $<2.0$ and the group of healthy subjects (Fig. 4b-c and Table 2). Furthermore, MFS patients with dilated pDAo also showed lower $\mathrm{WSS}_{\text {mean }}$ at the inner pAAo segment $(p<0.05)$ and outer dDAo segment $(p<0.05)$ compared to healthy subjects.

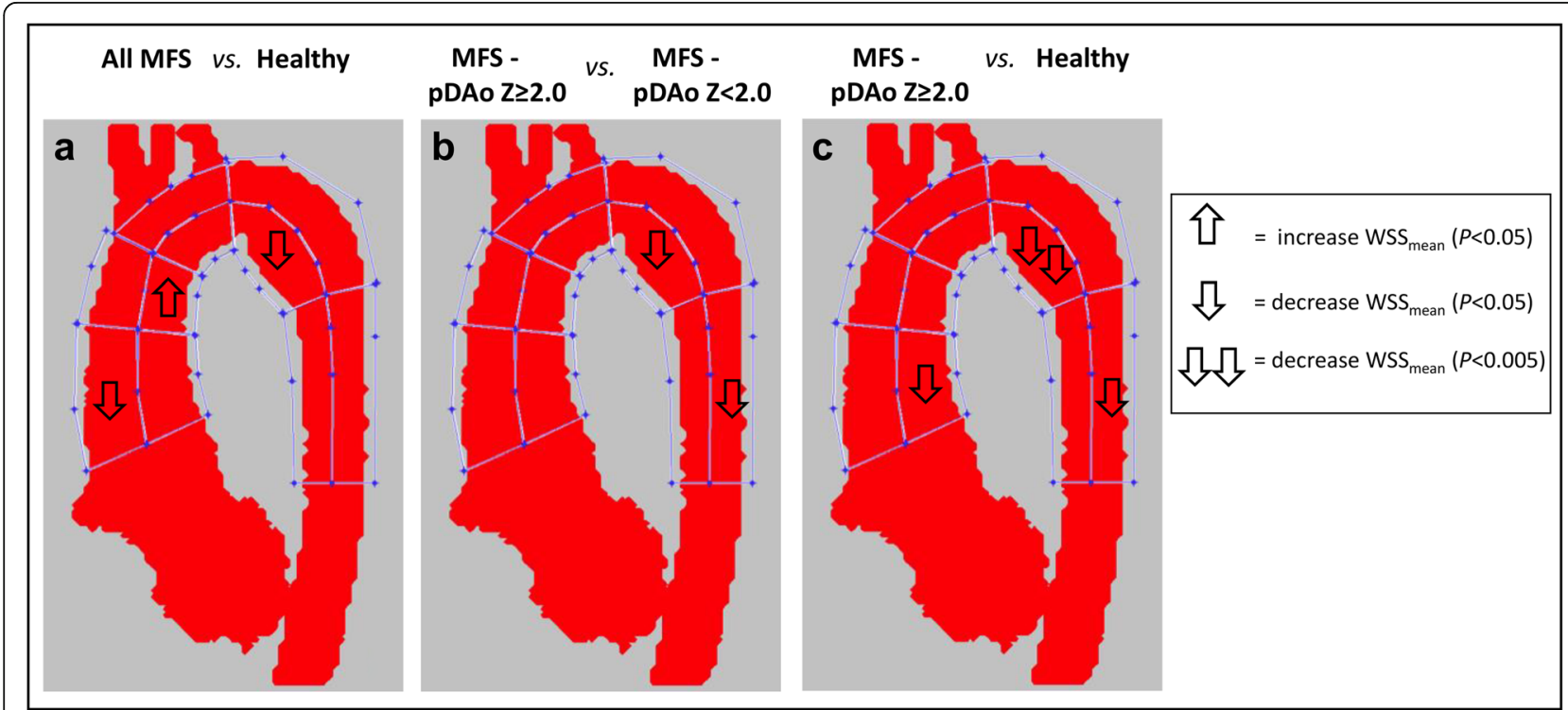

Fig. 4 Comparison of mean systolic wall shear stress in the ten aortic regions between groups. a entire MFS cohort $(n=25)$ vs. healthy subjects $(n=21)$. b MFS with a pDAo Z-score $\geq 2.0(n=7)$ vs. MFS with a pDAo Z-score $<2.0(n=18)$. c MFS with pDAo Z-score $\geq 2.0(n=7)$ vs. healthy subjects $(n=21)$ 


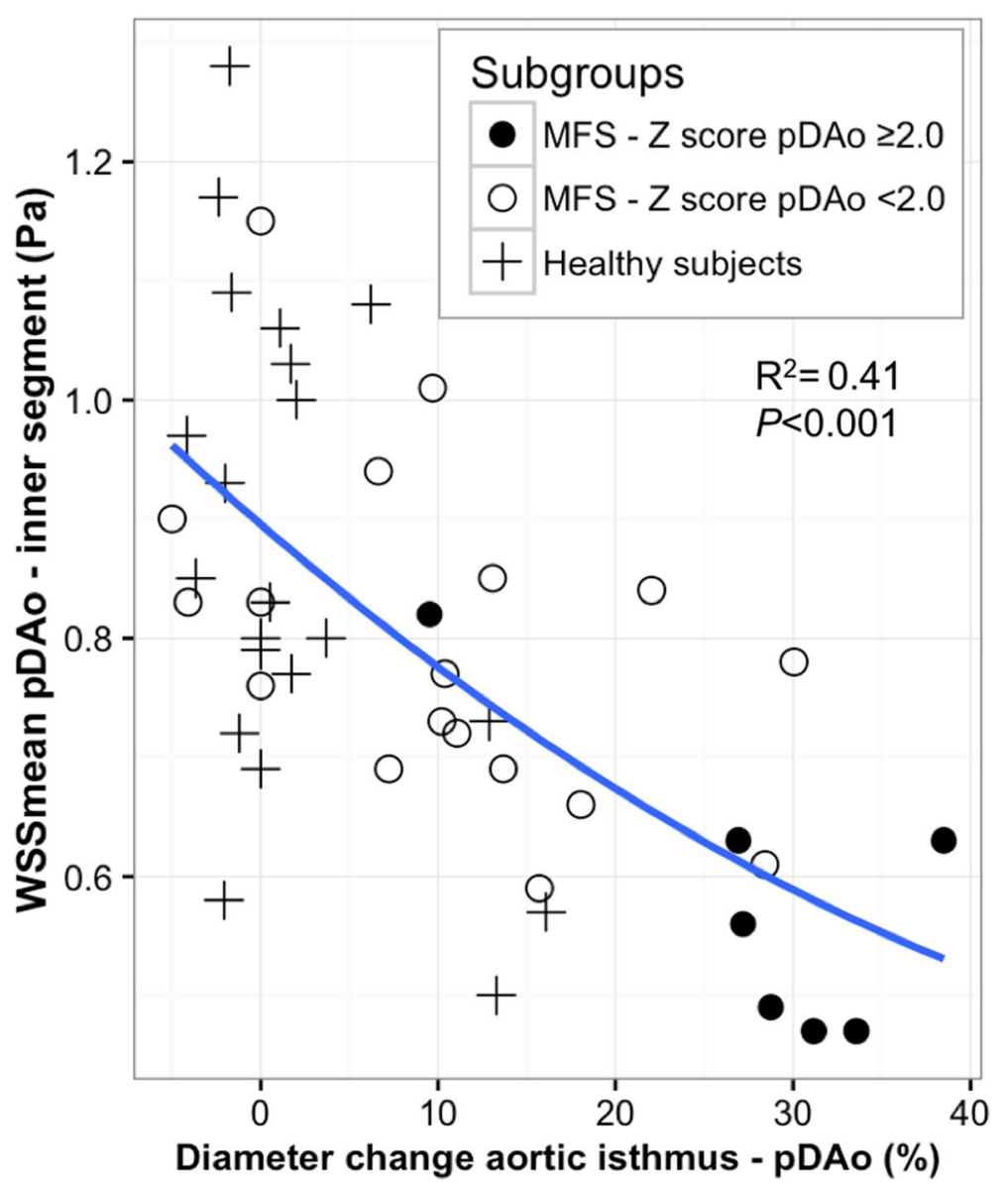

Fig. 5 Relationship between size change and inner wall shear stress in the proximal descending aortic segment. Inverse relation between diameter change between aortic isthmus and pDAo segment (in \% of isthmus diameter) and WSS mean of the pDAo segment in MFS patients and healthy subjects

\section{Discussion}

The findings of this study demonstrate that pediatric MFS patients have significantly lower WSS $_{\text {mean }}$ in the pAAo and pDAo than age-matched healthy controls. These regions in the aorta correspond to the locations where aortic dissection and aortic rupture often originate $[2,4,5]$. The subgroup of MFS patients with already dilated pDAo showed more prominent local vortex flow patterns and markedly lower $\mathrm{WSS}_{\text {mean }}$ in the dilated pDAo region, when compared to non-dilated pDAo MFS patients and healthy controls. Furthermore, an inverse relationship between regional aortic diameter and WSS $_{\text {mean }}$ was observed in the entire study group.

Aortic dilatation in MFS patients may start in early childhood, warranting close clinical follow up of the diameters in the entire thoracic aorta from the time of diagnosis. The average aortic size and the heterogeneous involvement of the pDAo regarding dilatation found in this study are in concordance with previous studies [27, 28]. Although MFS is known to be prone to ascending aortic complications, a substantial part of the aortic dissections in MFS occur in the
pDAo before reaching surgical cut-off levels. European studies reported the pDAo to be the site of the first aortic complications in $16-18 \%$ of the MFS patients $[2,29]$. This proportion will increase after elective surgery of the AAo [4, 5]. Changes in WSS in the aorta caused by changes in flow may be the key to better understand aortic disease development in MFS.

\section{Aortic flow patterns and wall shear stress}

Mechanisms leading to progressive aortic dilatation and elongation are not fully understood. Recently, aortic flow and derived hemodynamic factors from CMR, including WSS, have been associated with focal evidence of molecular and architectural tissue dysfunction in patients with bicuspid aortic valve and aortopathy [15]. This study confirms that children and adolescent MFS patients already have disturbed aortic flow patterns in the thoracic aorta. The regions of prominent flow alterations were the aortic root and pDAo, with a significantly higher prevalence and strength of vortex flow patterns found in the pDAo in MFS compared to healthy 
Table 2 Comparison of aortic dimension and WSS between MFS groups and healthy subjects

\begin{tabular}{|c|c|c|c|}
\hline & $\begin{array}{l}\text { Group } 1 \\
\text { MFS - pDAo Z } \geq 2.0 \\
(n=7)\end{array}$ & $\begin{array}{l}\text { Group } 2 \\
\text { MFS - pDAo } Z<2.0 \\
(n=18)\end{array}$ & $\begin{array}{l}\text { Group } 3 \\
\text { Healthy subjects } \\
(n=21)\end{array}$ \\
\hline Age (y) & $15.6 \pm 4.5$ & $15.6 \pm 4.0$ & $16.0 \pm 2.6$ \\
\hline Females $(n)$ & 3 & 8 & 12 \\
\hline $\mathrm{BSA}\left(\mathrm{m}^{2}\right)$ & $1.6 \pm 0.4$ & $1.8 \pm 0.3$ & $1.6 \pm 0.3$ \\
\hline \multicolumn{4}{|l|}{ Thoracic aortic size (Z-score) } \\
\hline Z-score Aortic Root** & $4.4 \pm 1.3$ & $3.2 \pm 1.4$ & $0.5 \pm 0.8$ \\
\hline Z-score STJ** & $3.0 \pm 0.9$ & $1.2 \pm 1.0$ & $-0.3 \pm 0.8$ \\
\hline Z-score $A A o^{* *}$ & $1.1 \pm 0.7$ & $-0.1 \pm 0.8$ & $-0.5 \pm 0.6$ \\
\hline Z-score distal Arch* & $0.8 \pm 0.7$ & $-0.1 \pm 0.9$ & $-0.2 \pm 0.7$ \\
\hline Z-score Isthmus ${ }^{* *}$ & $2.0 \pm 0.7$ & $0.6 \pm 0.7$ & $0.4 \pm 0.5$ \\
\hline Z-score proximal DAo** & $4.2 \pm 1.1$ & $1.2 \pm 0.6$ & $0.6 \pm 0.7$ \\
\hline$\Delta$ diameter Root-AAo $(\%)^{* *}$ & $30.9 \pm 4.0$ & $33.8 \pm 7.0$ & $19.0 \pm 7.0$ \\
\hline$\Delta$ diameter Isthmus-pDAo $(\%)^{* *}$ & $27.9 \pm 9.1$ & $10.4 \pm 10.1$ & $1.9 \pm 5.7$ \\
\hline \multicolumn{4}{|l|}{ WSS mean $(\mathrm{Pa})$} \\
\hline 1. Inner pAAo* & $0.69 \pm 0.18$ & $0.81 \pm 0.09$ & $0.86 \pm 0.16$ \\
\hline 2. Outer pAAo & $0.59 \pm 0.11$ & $0.67 \pm 0.11$ & $0.73 \pm 0.14$ \\
\hline 3. Inner dAAo* & $0.85 \pm 0.18$ & $0.97 \pm 0.11$ & $0.85 \pm 0.15$ \\
\hline 4. Outer dAAo & $0.75 \pm 0.14$ & $0.87 \pm 0.11$ & $0.83 \pm 0.14$ \\
\hline 5. Inner Arch & $0.83 \pm 0.13$ & $0.99 \pm 0.13$ & $0.91 \pm 0.16$ \\
\hline 6. Outer Arch & $0.71 \pm 0.09$ & $0.80 \pm 0.11$ & $0.79 \pm 0.14$ \\
\hline 7. Inner pDAo* & $0.58 \pm 0.13$ & $0.80 \pm 0.14$ & $0.87 \pm 0.21$ \\
\hline 8. Outer pDAo & $0.76 \pm 0.13$ & $0.89 \pm 0.10$ & $0.86 \pm 0.12$ \\
\hline 9. Inner dDAo & $0.75 \pm 0.09$ & $0.89 \pm 0.16$ & $0.89 \pm 0.17$ \\
\hline 10. Outer dDAo* & $0.78 \pm 0.05$ & $0.99 \pm 0.18$ & $0.97 \pm 0.16$ \\
\hline
\end{tabular}

Significant across subgroups using a Kruskal-Wallis test. ${ }^{*} p<0.05,{ }^{* *} p<0.001$. Abbreviations: $p A A o$ proximal ascending aorta, dAAo distal ascending aorta, Arch aortic arch, $p D A o$ proximal descending aorta, $d D A o$ distal descending aorta

subjects. Although it is known that small vortex flow patterns in the pDAo sometimes occur in normal aortas with an aortic spindle (i.e. a normal variation in the aortic contour not to be confused with an aneurysm) [30], the high prevalence and extensive nature found in these young MFS patients in this study is remarkable and consistent with observations by two previous CMR studies in MFS, with prevalence rates ranging from 54 to $70 \%$ $[8,31]$. Local aortic geometry seems to be related to the formation of these flow phenomena as the MFS subjects with most dilated pDAo segments (Z-score $\geq 2.0$ ) showed the most extensive focal vortex flow patterns in this study.

Several studies previously demonstrated that visibly pathologic flow patterns could lead to measurable alterations in WSS [32, 33]. Interestingly, the observed vortex flow patterns in the pDAo in MFS patients in this study corresponded to the reduced WSS mean $_{\text {observed in the }}$ same segment. A similar relationship has been described in a longitudinal follow-up study of MFS patients after root replacement where vortex flow disturbances within the pDAo segment together with reduced local WSS measures developed [34]. In addition to the lower $\mathrm{WSS}_{\text {mean }}$ in the pDAo segment, considerable differences in WSS $_{\text {mean }}$ were observed in the pAAo outer segment of the MFS patients compared to healthy subjects. However, no obvious flow pattern differences in the AAo were detected between MFS patients and the controls, except for local helix formation originating from the aortic root into the proximal AAo in four MFS patients. The significant difference in AAo size between the MFS and healthy controls could have contributed to this reduced regional WSS $_{\text {mean }}$.

Few studies have investigated aortic WSS in MFS disease cohorts so far $[9,10]$ and comparable investigations on aortic WSS were conducted in similar patients groups with dilative aortopathy of unknown origin (i.e. non-bicuspid valve related diseases) [32, 35]. As in MFS patients, these patients have in common that the AAo is more or less dilated and these studies revealed similar aortic WSS results. In general, lower systolic WSS values were found along the dilated thoracic aorta in both these patient groups, as provided by $2 \mathrm{D}$ planar WSS 
quantification methods $[9,32,35]$. Similar to our findings, the specific locations of reduced systolic WSS were found at the outer curvature of the pAAo in most of these studies $[9,35]$. Contrary to our results, one of the WSS studies in pediatric MFS patients showed inhomogeneous but relatively higher peak systolic WSS values at focal points on the vessel lumen circumference in the AAo compared to a healthy control group [10]. Although this is inconsistent to our findings, the higher WSS found in that study might be explained by the fact that the MFS patients were younger and had similar or even significantly smaller indexed aortic dimensions compared to the older healthy control group. Indeed, both factors (i.e. younger age and smaller vessel dimensions) have been proven to be associated with relatively higher WSS measures [36, 37]. The pDAo WSS in the MFS cohorts from these 4D flow CMR studies was equal or lower in MFS, but statistically similar compared to the WSS of healthy controls $[9,10]$. It should be noted that the aortic WSS measurements in these comparative studies were all based on 2D planar WSS quantification methods, which means that WSS measurements are derived from single 2D cross-sectional planes along the thoracic aorta.

In this study, we used a novel approach providing 3D WSS along the entire aortic wall that was recently developed $[25,26]$. The major advantage of this 3D WSS approach is that the entire aorta is included rather than sampling only a fraction of the vessel wall, thus reducing the chance of missing important regional variations in WSS. Moreover, it allows regional comparison to control references and no manual placement of 2D crosssectional planes is needed, which is more subject to observer variability. In addition, the estimation of 3D WSS over a certain regional vessel surface enables to study relationships between regional hemodynamics and geometric measures, for instance aortic diameter or diameter changes between vessel segments.

\section{Aortic WSS and diameter}

The findings of our study revealed a close interaction between altered aortic diameter and regional $\mathrm{WSS}_{\text {mean }}$ in MFS patients and healthy subjects. First, an inverse relationship between $\mathrm{WSS}_{\text {mean }}$ and aortic diameter was observed, which is congruent with existing literature $[35,36,38]$. The most obvious correlation was found for the pDAo segment, where both diameter and diameter change between the aortic isthmus and the largest pDAo dimension were inversely proportional to $\mathrm{WSS}_{\text {mean }}$. Furthermore, subgroup analysis of MFS patients with a dilated pDAo showed the lowest $\mathrm{WSS}_{\text {mean }}$ in the corresponding region. Second, the dilation of the aortic root and STJ in MFS patients may have resulted in reduced $\mathrm{WSS}_{\text {mean }}$ values in the
pAAo segment and might explain the $\mathrm{WSS}_{\text {mean }}$ asymmetry between the inner and outer pAAo segments. Such WSS asymmetry was also observed in the pAAo segment in another 4D flow CMR study on MFS patients [9]. Moreover, an in-vitro computational fluid dynamic (CFD) investigation about the effect of morphological changes in the aortic root on flow hemodynamics supports these findings. That CFD study, which was conducted with MFS-specific aortic root models of increasing size, showed that progressive root dilatation resulted in a different orientation of the blood stream jet in the aortic root and pAAo [39]. It is conceivable that a change in flow displacement from the sinus side towards the commissure side has resulted in the altered distribution of WSS $_{\text {mean }}$ that was observed in the pAAo in the MFS patients of this study.

\section{Clinical perspectives}

Current management strategies for preventive aortic replacement in MFS are based on aortic diameters or rate of dilatation. However, this strategy remains imperfect and has led to a search for additional markers of disease severity and predictors of adverse vascular events, such as hemodynamic parameters. The results of this study showed already hemodynamic differences in young MFS patients compared to age-appropriate healthy subjects and determined close relationships between regional aortic geometry, flow patterns and 3D WSS over certain vessel regions. This relationship was most evident for the pDAo. Whether these hemodynamic parameters (i.e. flow and WSS) may add in the prediction of progressive dilatation, adverse events or optimization of management strategies in future in addition to the current diameter measurements is unknown and requires further longitudinal follow-up. In this regard and in line with our findings, a recent longitudinal 4D flow follow-up study on MFS patients after preventive valve-sparing aortic root replacement reported the occurrence of helical and vortex flow pattern formation along the inner curvature of the pDAo in $50 \%$ of the patients [34]. Additionally, in that report, a single case was described with newly derived regional vortex formation in the pDAo and altered WSS in that region prior to the development of an intramural hematoma with subsequent aortic dissection type B in the pDAo. This occurred 5 years after root replacement and the changes in flow and WSS parameters are noteworthy and suggest that these factors may play a role in the onset of the adverse event [34]. Together with our results, these observations show the clinical potential and future perspectives of comprehensive aortic flow hemodynamics assessments and monitoring of longitudinal changes by $4 \mathrm{D}$ flow imaging for optimization of preventive decision making. 
Based on these and our data, one might speculate that at least the MFS patients with pDAo Z-score $\geq 2.0$, abnormal vortex flow patterns and decreased local WSS mean $_{\text {, }}$ are at higher risk for adverse cardiovascular events. Additional longitudinal studies are warranted to further elucidate the diagnostic value of inter-individual differences in MFS hemodynamics and the applicability of 4D flow parameters in prediction models.

\section{Study limitations}

The current study is subject to several limitations. In this observational study there might be some selection bias. Although MFS patients underwent CMR as a regular part of their lifelong clinical follow-up according to the MFS imaging guidelines, we cannot completely exclude the possibility that some of them received MR imaging based on previously abnormal findings on echocardiographic imaging. These results therefore may not be generalizable. However, it is known that the MFS patients individually can show a large heterogeneity in the involvement of heart and cardiovascular problems, e.g. the onset and the progression of disease over time.

To account for patient age and body size for aortic dimensions comparison in children, aortic Z-scores were calculated using EchoIMS. While EchoIMS provides ultrasoundderived normative data, there is currently no large pediatric CMR-specific database and this method of normalization is the best available alternative, is used in clinical practice and has been applied in previous CMR studies [22, 23].

Finally, potential sources of error, including the limited spatial resolution of $4 \mathrm{D}$ flow CMR, could have resulted in underestimation of $3 \mathrm{D} \mathrm{WSS}_{\text {mean }}$ measurements. However, as both patient and healthy control groups underwent MR imaging at the same scanners with similar scan parameters, relative differences in $\mathrm{WSS}_{\text {mean }}$ between the two groups remain useful. Furthermore, 3D segmentation of the aorta based on the time-averaged systolic portion of the cardiac cycle is a potential source of error in this 4D flow study. However, averaging WSS over five systolic phases of the cardiac cycle with our algorithm, and using a well-defined segmentation protocol have been proven to produce low variability [26].

\section{Conclusions}

MFS children and young adults have altered aortic flow patterns and differences in WSS that were most pronounced in the pAAo and pDAo, which correspond to the locations where aortic dissection and aortic rupture often originate in these patients. Furthermore, close relationships between the regional aortic size and the presence of abnormal flow patterns and WSS were demonstrated and were more evident in the pDAo segment. These findings indicate that hemodynamic parameters may be discriminative and potentially valuable additional markers of disease severity. Additional longitudinal studies correlating changes in hemodynamic parameters (aortic flow profiles and WSS) with aortopathy are needed to establish the role of these parameters in disease progression and risk prediction of adverse events in MFS patients.

\section{Additional files}

Additional file 1: Video S1. Pathline visualization from a MFS patient's aorta with local vortex flow pattern in the proximal descending aorta during systole and early diastole. Note the prominent vortex flow patterns in the sinuses of the aortic root. (MPG $3532 \mathrm{~kb}$ )

Additional file 2: Video S2. Pathline visualization of a normal aorta from a healthy subject. No local flow pattern abnormalities are present. (MPG 2044 kb)

\section{Abbreviations}

2D: Two-dimensional; 3D: Three-dimensional; 4D flow CMR: Three-dimensional phase-contrast CMR with three-directional velocity encoding; AAo: Ascending aorta; CE-MRA: Contrast-enhanced MR angiography; CFD: Computational fluid dynamic; CMR: Cardiovascular magnetic resonance; dAAo: Distal ascending aorta; DAo: Descending aorta; dDAo: Distal descending aorta; FBN1: Fibrillin-1; IRB: Institutional Review Board; MFS: Marfan syndrome; midAAo: Mid-ascending aorta; midDAo: Mid-descending aorta; pAAo: Proximal ascending aorta; pDAo: Proximal descending aorta; SSFP: Steady state free precession; STJ: Sinotubular junction; Vel: Velocity; WSS: Wall shear stress; WSS mean: 3D mean systolic wall shear stress

\section{Acknowledgements}

Not applicable.

\section{Funding}

This work was supported by the Netherlands Heart Foundation grant 2014 T087, The Royal Netherlands Academy of Arts and Sciences - Academy Ter Meulen grant 15/275, National Institutes of Health grants R01HL115828 and K25HL119608 and American Heart Association grants 14POST18350019 and 16POST27250158.

\section{Availability of data and material}

The datasets used and/or analysed during the current study available from the corresponding author on reasonable request.

\section{Authors contributions}

$\mathrm{RP}$, conception and design of study, methods development, data analysis and interpretation of data, drafting of manuscript and revision. EB, JG: methods development, data analysis and interpretation of data, revision of manuscript. AR, LY: interpretation of data, drafting of manuscript and revision. MR: coordination of data collection, methods development, data analysis, revision of manuscript. $A B, J R, M M, C R$ : conception and design of study, coordination of data collection and interpretation of data, drafting of manuscript and revision. All authors read and approved the final manuscript.

Competing of interests

The authors declare that they have no competing interests.

\section{Consent for publication}

All participants in this study gave written consent to participate and to publish.

\section{Ethics approval and consent to participate}

The study was approved by the institutional review board of the Ann \& Robert H. Lurie Children's Hospital and Northwestern University. Written informed consent was obtained from all participants, their parents or legal guardians to participate in the study, including the addition of the 4D flow CMR to the standard-of-care cardiac CMR protocol. 


\section{Author details}

'Department of Radiology, Feinberg School of Medicine, Northwestern University , Chicago, IL, USA. 'Division of Pediatric Cardiology, Department of Pediatrics, Leiden University Medical Center, Albinusdreef 2, 2333, ZA, Leiden, The Netherlands. ${ }^{3}$ Department of Cardiac Sciences, Stephenson Cardiac Imaging Centre, University of Calgary - Cumming School of Medicine, Calgary, AB, Canada. ${ }^{4}$ Department of Medical Imaging, Ann \& Robert H. Lurie Children's Hospital of Chicago, Chicago, IL, USA. ${ }^{5}$ Department of Radiology, Academic Medical Center, Amsterdam, The Netherlands. ${ }^{6}$ Department of Biomedical Engineering, McCormick School; of Engineering, Northwestern University, Chicago, IL, USA. 'Department of Pediatrics, Division of Pediatric Cardiology, Ann \& Robert H. Lurie Children's Hospital of Chicago, Chicago, IL, USA. ${ }^{8}$ Department of Pediatrics, Feinberg School of Medicine, Northwestern University, Chicago, IL, USA.

Received: 24 November 2016 Accepted: 16 February 2017

Published online: 17 March 2017

\section{References}

1. Alpendurada F, Mohiaddin R. Prevalence of cardiovascular manifestations in patients with Marfan syndrome: a cardiovascular magnetic resonance stud. J Cardiovasc Magn Reson. 2008;10 Suppl 1:A164

2. Engelfriet PM, Boersma E, Tijssen JG, Bouma BJ, Mulder BJ. Beyond the root: dilatation of the distal aorta in Marfan's syndrome. Heart. 2006;92:1238-43.

3. Mariucci EM, Lovato L, Rosati M, Palena LM, Bonvicini M, Fattori R. Dilation of peripheral vessels in Marfan syndrome: importance of thoracoabdominal MR angiography. Int J Cardiol. 2013;167:2928-31.

4. Mimoun L, Detaint D, Hamroun D, Arnoult F, Delorme G, Gautier M, Milleron O, Meuleman C, Raoux F, Boileau C, et al. Dissection in Marfan syndrome: the importance of the descending aorta. Eur Heart J. 2011;32:443-9.

5. den Hartog AW, Franken $R$, Zwinderman AH, Timmermans J, Scholte AJ, van den Berg MP, de Waard V, Pals G, Mulder BJ, Groenink M. The risk for type B aortic dissection in Marfan syndrome. J Am Coll Cardiol. 2015;65:246-54.

6. Erbel R, Aboyans V, Boileau C, Bossone E, Bartolomeo RD, Eggebrecht $\mathrm{H}$, Evangelista A, Falk V, Frank H, Gaemperli O, et al. ESC Guidelines on the diagnosis and treatment of aortic diseases: document covering acute and chronic aortic diseases of the thoracic and abdominal aorta of the adult. The Task Force for the Diagnosis and Treatment of Aortic Diseases of the European Society of Cardiology (ESC). Eur Heart J. 2014;2014(35):2873-926.

7. van Ooij P, Potters W, Collins J, Carr M, Carr J, Malaisrie SC, Fedak PW, McCarthy PM, Markl M, Barker AJ. Characterization of abnormal wall shear stress using 4D flow MRI in human bicuspid aortopathy. Ann Biomed Eng. 2015:43:1385-97.

8. Geiger J, Markl M, Herzer L, Hirtler D, Loeffelbein F, Stiller B, Langer M, Arnold R. Aortic flow patterns in patients with Marfan syndrome assessed by flowsensitive four-dimensional MRI. J Magn Reson Imaging. 2012:35:594-600.

9. Wang $\mathrm{HH}$, Chiu HH, Tseng Wl, Peng HH. Does altered aortic flow in marfan syndrome relate to aortic root dilatation? J Magn Reson Imaging. 2016:44:500-08.

10. Geiger J, Arnold R, Herzer L, Hirtler D, Stankovic Z, Russe M, Langer M, Markl M. Aortic wall shear stress in Marfan syndrome. Magn Reson Med. 2013:70:1137-44.

11. Wigstrom L, Ebbers T, Fyrenius A, Karlsson M, Engvall J, Wranne B, Bolger AF. Particle trace visualization of intracardiac flow using time-resolved 3D phase contrast MRI. Magn Reson Med. 1999;41:793-9.

12. Buonocore $\mathrm{MH}$. Visualizing blood flow patterns using streamlines, arrows, and particle paths. Magn Reson Med. 1998;40:210-26.

13. Markl M, Frydrychowicz A, Kozerke S, Hope M, Wieben O. 4D flow MRI. J Magn Reson Imaging. 2012;36:1015-36.

14. Markl M, Schnell S, Wu C, Bollache E, Janvis K, Barker AJ, Robinson JD, Rigsby CK. Advanced flow MRl: emerging techniques and applications. Clin Radiol. 2016;71: 779-95.

15. Guzzardi DG, Barker AJ, van Ooij P, Malaisrie SC, Puthumana JJ, Belke DD, Mewhort HE, Svystonyuk DA, Kang S, Verma S, et al. Valve-related hemodynamics mediate human bicuspid aortopathy: insights from wall shear stress mapping. J Am Coll Cardiol. 2015;66:892-900

16. Loeys BL, Dietz HC, Braverman AC, Callewaert BL, De Backer J, Devereux RB, Hilhorst-Hofstee $Y$, Jondeau G, Faivre L, Milewicz DM, et al. The revised Ghent nosology for the Marfan syndrome. J Med Genet. 2010;47:476-85.

17. Hiratzka LF, Bakris GL, Beckman JA, Bersin RM, Carr VF, Casey Jr DE, Eagle KA, Hermann LK, Isselbacher EM, Kazerooni EA, et al. ACCF/AHA/ AATS/ACR/ASA/SCA/SCAI/SIR/STS/SVM Guidelines for the diagnosis and management of patients with thoracic aortic disease. A Report of the American College of Cardiology Foundation/American Heart Association Task Force on Practice Guidelines, American Association for Thoracic Surgery, American College of Radiology,American Stroke Association, Society of Cardiovascular Anesthesiologists, Society for Cardiovascular Angiography and Interventions, Society of Interventional Radiology, Society of Thoracic Surgeons,and Society for Vascular Medicine. J Am Coll Cardiol. 2010;55:e27-e129.

18. Burman ED, Keegan J, Kilner PJ. Aortic root measurement by cardiovascular magnetic resonance: specification of planes and lines of measurement and corresponding normal values. Circ Cardiovasc Imaging. 2008;1:104-13.

19. Nejatian A, Yu J, Geva T, White MT, Prakash A. Aortic measurements in patients with aortopathy are larger and more reproducible by cardiac magnetic resonance compared with echocardiography. Pediatr Cardiol. 2015:36:1761-73.

20. Mosteller RD. Simplified calculation of body-surface area. N Engl J Med. 1987;317:1098.

21. Chubb H, Simpson JM. The use of Z-scores in paediatric cardiology. Ann Pediatr Cardiol. 2012:5:179-84.

22. Johnson RK, Premraj S, Patel SS, Wahle A, Stolpen A, Sonka M, Scholz TD. Quantitative assessment of the entire thoracic aorta from magnetic resonance images. Cardiol Young. 2011;21:170-7.

23. Allen BD, van Ooij P, Barker AJ, Carr M, Gabbour M, Schnell S, Jarvis KB, Carr JC, Markl M, Rigsby C, Robinson JD. Thoracic aorta 3D hemodynamics in pediatric and young adult patients with bicuspid aortic valve. J Magn Reson Imaging. 2015;42:954-63.

24. Bock J, Kreher B, Henning J, Markl M. Optimized pre-processing of timeresolved $2 \mathrm{D}$ and $3 \mathrm{D}$ phase contrast MRI data. Proceedings of the 15 th Annual Meeting of ISMRM, Berlin, Germany. 2007:604. (Abstract 3138).

25. Potters W, van Ooii $P$, Marquering $H$, van Bavel E, Nederveen AJ. Volumetric arterial wall shear stress calculation based on cine phase contrast MRI. J Magn Reson Imaging. 2015:41:505-16.

26. van Ooij P, Powell AL, Potters W, Carr JC, Markl M, Barker AA. Reproducibility and interobserver variability of systolic blood flow velocity and 3D wall shear stress derived from 4D flow MRI in the healthy aorta. J Magn Reson Imaging. 2016:43:236-48.

27. Faivre L, Masurel-Paulet A, Collod-Beroud G, Callewaert BL, Child AH, Stheneur C, Binquet C, Gautier E, Chevallier B, Huet F, et al. Clinical and molecular study of 320 children with Marfan syndrome and related type I fibrillinopathies in a series of 1009 probands with pathogenic FBN1 mutations. Pediatrics. 2009:123:391-8.

28. van Karnebeek CD, Naeff MS, Mulder BJ, Hennekam RC, Offringa M. Natural history of cardiovascular manifestations in Marfan syndrome. Arch Dis Child. 2001;84:129-37

29. Finkbohner R, Johnston D, Crawford ES, Coselli J, Milewicz DM. Marfan syndrome. Long-term survival and complications after aortic aneurysm repair. Circulation. 1995:91:728-33.

30. Fisher RG, Sanchez-Torres M, Whigham CJ, Thomas JW. "Lumps" and "bumps" that mimic acute aortic and brachiocephalic vessel injury. Radiographics. 1997;17:825-34.

31. Picher A, Cassar TE, Suttie J, Francis JM, Leeson P, Blair E, Wordsworth BP, Forfar JC, Myerson SG, Markl M, et al. Visualisation of aortic flow disturbance in Marfan syndrome by 4D phase-contrast CMR. J Cardiovasc Magn Reson. 2011;13 Suppl 1:201.

32. Bieging ET, Frydrychowicz A, Wentland A, Landgraf BR, Johnson KM, Wieben O, Francois CJ. In vivo three-dimensional MR wall shear stress estimation in ascending aortic dilatation. J Magn Reson Imaging. 2011; 33:589-97.

33. Frydrychowicz A, Arnold R, Hirtler D, Schlensak C, Stalder AF, Hennig J, Langer M, Markl M. Multidirectional flow analysis by cardiovascular magnetic resonance in aneurysm development following repair of aortic coarctation. J Cardiovasc Magn Reson. 2008;10:30.

34. Hope TA, Kvitting JP, Hope MD, Miller DC, Markl M, Herfkens RJ. Evaluation of Marfan patients status post valve-sparing aortic root replacement with 4D flow. Magn Reson Imaging. 2013;31:1479-84

35. Burk J, Blanke P, Stankovic Z, Barker A, Russe M, Geiger J, Frydrychowicz A, Langer M, Markl M. Evaluation of 3D blood flow patterns and wall shear stress in the normal and dilated thoracic aorta using flow-sensitive 4D CMR. J Cardiovasc Magn Reson. 2012;14:84.

36. Truong U, Fonseca B, Dunning J, Burgett S, Lanning C, Ivy DD, Shandas R Hunter K, Barker AJ. Wall shear stress measured by phase contrast 
cardiovascular magnetic resonance in children and adolescents with pulmonary arterial hypertension. J Cardiovasc Magn Reson. 2013;15:81

37. van Ooij P, Garcia J, Potters WW, Malaisrie SC, Collins JD, Carr JC, Markl M, Barker AJ. Age-related changes in aortic 3D blood flow velocities and wall shear stress: Implications for the identification of altered hemodynamics in patients with aortic valve disease. J Magn Reson Imaging. 2016:43:1239-49.

38. Allen BD, Markl M, Barker AJ, van Ooij P, Carr JC, Malaisrie SC, McCarthy P, Bonow RO, Kansal P. Influence of beta-blocker therapy on aortic blood flow in patients with bicuspid aortic valve. Int J Cardiovasc Imaging. 2016;32:621-8.

39. Querzoli G, Fortini S, Espa S, Costantini M, Sorgini F. Fluid dynamics of aortic root dilation in Marfan syndrome. J Biomech. 2014:47:3120-8.

Submit your next manuscript to BioMed Central and we will help you at every step:

- We accept pre-submission inquiries

- Our selector tool helps you to find the most relevant journal

- We provide round the clock customer support

- Convenient online submission

- Thorough peer review

- Inclusion in PubMed and all major indexing services

- Maximum visibility for your research

Submit your manuscript at www.biomedcentral.com/submit
Biomed Central 